\title{
Successful optic nerve regeneration in the senescent zebrafish despite age-related decline of cell intrinsic and extrinsic response processes
}

\author{
Jessie Van houcke ${ }^{\mathrm{a}, 1}$, Ilse Bollaerts ${ }^{\mathrm{a}, 1}$, Emiel Geeraerts ${ }^{\mathrm{a}}$, Benjamin Davis ${ }^{\mathrm{b}}$, An Beckers ${ }^{\mathrm{a}}$, \\ Inge Van Hove ${ }^{a}$, Kim Lemmens ${ }^{a}$, Lies De Groef ${ }^{a, b}$, Lieve Moons ${ }^{a, *}$ \\ ${ }^{a}$ Laboratory of Neural Circuit Development and Regeneration, Animal Physiology and Neurobiology Section, Department of Biology, KU Leuven, Leuven, \\ Belgium \\ ${ }^{\mathrm{b}}$ Glaucoma and Retinal Neurodegeneration Research, Visual Neuroscience, UCL Institute of Ophthalmology, London, UK
}

\section{A R T I C L E I N F O}

\section{Article history:}

Received 19 April 2017

Received in revised form 18 July 2017

Accepted 13 August 2017

Available online 24 August 2017

\section{Keywords:}

Aging

Zebrafish

Axonal regeneration

Central nervous system

Cellular senescence

Inflammation

\begin{abstract}
A B S T R A C T
Dysfunction of the central nervous system (CNS) in neurodegenerative diseases or after brain lesions seriously affects life quality of a growing number of elderly, since the adult CNS lacks the capacity to replace or repair damaged neurons. Despite intensive research efforts, full functional recovery after CNS disease and/or injury remains challenging, especially in an aging environment. As such, there is a rising need for an aging model in which the impact of aging on successful regeneration can be studied. Here, we introduce the senescent zebrafish retinotectal system as a valuable model to elucidate the cellular and molecular processes underlying age-related decline in axonal regeneration capacities. We found both intrinsic and extrinsic response processes to be altered in aged fish. Indeed, expression levels of growthassociated genes are reduced in naive and crushed retinas, and the injury-associated increase in innate immune cell density appears delayed, suggesting retinal inflammaging in old fish. Strikingly, however, despite a clear deceleration in regeneration onset and early axon outgrowth leading to an overall slowing of optic nerve regeneration, reinnervation of the optic tectum and recovery of visual function occurs successfully in the aged zebrafish retinotectal system.
\end{abstract}

(c) 2017 Elsevier Inc. All rights reserved.

\section{Introduction}

Considering the increasing number of elderly in the world's population today, developing effective treatments for neurodegenerative diseases is one of the biggest challenges in modern medical research. Currently, their irreversibility and lack of effective treatments impinge a high social and economical burden and urge the development of therapeutic strategies to ameliorate and/or resolve neuropathology. This could in part be achieved by triggering endogenous stem cells to form new neurons, that is, neuronal regeneration, or protect damaged cells/axons from undergoing cell death, that is, neuroprotection. In both cases, new and/or injured neurons have to regrow a functional axon and restore synaptic contacts, thereby establishing axonal regeneration. Yet, regenerative capacities are very limited in the adult mammalian central

\footnotetext{
* Corresponding author at: Laboratory of Neural Circuit Development and Regeneration, Animal Physiology and Neurobiology Section, Department of Biology, Zoological Institute, Naamsestraat 61, 3000 Leuven, Belgium. Tel.: +32 16323991 ; fax: +32 16324262 .

E-mail address: lieve.moons@kuleuven.be (L. Moons).

1 These authors contributed equally to this work.
}

nervous system (CNS), and they are affected by age. Indeed, studies in rodents have shown that the age at the time of neuronal damage determines neuronal survival rate (Wang et al., 2007) as well as axonal regrowth potential (Geoffroy et al., 2016). As such, most research into mammalian CNS regeneration using young animal models might eventually fail successful translation into therapeutic approaches applicable in an aging environment. Clearly, wellcharacterized aging models, in which regeneration can be studied in a senescent environment, are highly needed.

Zebrafish (Danio rerio) are one of the most versatile animal research models. Their robust regenerative capacity continues to drive comparative research, and a remarkably high similarity in the signaling pathways underlying CNS regeneration in mammals and zebrafish has already been unraveled (Becker and Becker, 2014; Elsaeidi et al., 2014; Fleisch et al., 2011; Wang and Jin, 2011). Now, they are also increasingly valued in gerontology, as it is recently established that zebrafish-like most vertebrates-are subject to processes of gradual aging (Gerhard, 2003; Gerhard and Cheng, 2002; Keller and Murtha, 2004; Kishi, 2011; Kishi et al., 2003; Van houcke et al., 2015). Indeed, also in these small teleosts, reparative capacities diminish with aging. Yet, while the neurogenic 
potential in the brain (Edelmann et al., 2013) and axonal regeneration capacities of the peripheral nervous system (Graciarena et al., 2014) are shown to decline with age, the underlying molecules/ pathways contributing to these phenomena remain elusive.

The visual system forms a powerful model to study neuronal survival and axonal regrowth in the CNS. It is relatively accessible, and both structurally and functionally well conserved among vertebrates. As in mammals, the zebrafish retina is a layered structure and projects visual signals towards the brain via the optic nerve (ON), containing the axons of the retinal ganglion cells (RGCs). However, in contrast to mammals, the adult zebrafish visual system shows a high reparative capacity. When the zebrafish ON is injured, a substantial part of the RGCs is able to survive and regenerate (McCurley and Callard, 2010; Zhou and Wang, 2002; Zou et al., 2013). After a short injury response, the RGCs start preparing for axonal outgrowth by upregulation of growth-associated genes from 1 day till 4 days postinjury (dpi) (Lemmens et al., 2016; McCurley and Callard, 2010). Subsequently, the RGC axons regrow towards the optic tectum, and reinnervation is completed between 5 and 14 dpi (Bhumika et al., 2015; Kaneda et al., 2008; Lemmens et al., 2016). Finally, full recovery of visual function is achieved by 20-25 dpi, although synaptic refinement of the retinotectal topography is known to continue until 30-90 days after injury (Becker and Becker, 2008; Kaneda et al., 2008; McCurley and Callard, 2010; Zou et al., 2013).

In this study, we examine the impact of aging on the axonal regeneration potential of the zebrafish retinotectal system and show that despite a significant delay in the initiation of axonal regeneration and early outgrowth, aged zebrafish are capable of successful $\mathrm{ON}$ regeneration. Indeed, regardless of age-related alterations in both intrinsic and extrinsic cellular response processes, (old) zebrafish can reestablish visual function after optic nerve crush (ONC).

\section{Material and methods}

\subsection{Fish}

Zebrafish (D. rerio) on an AB background, either wild-type (total of 127 fish younger and 106 fish older than 18 months) or the $\operatorname{Tg}$ (Gap-43:eGFP) (total of 23 5-month-old and 22 24-month-old fish) and $\mathrm{Tg}$ (coro1a:eGFP) (total of 32 5-month-old and 36 24-month-old fish) lines, were used and maintained under standard conditions (Westerfield, 2000). The ages chosen for ON regeneration analyses represent young and aged adults of $5,12,18$, 24 , and 36 months old. Both fish density and food supply were under strict regulation over the entire life span of the fish, resulting in uniform fish sizes within each age group. Fish size was measured as the standard length, that is, the distance from the snout to the base of the tail fin, and ranged from $29.67 \pm 1.12 \mathrm{~mm}$ in 5-monthold fish to $36.20 \pm 0.73 \mathrm{~mm}$ in 36-month-old individuals. Fish were randomized within the different experiments, with the exception of their sex parameter. Considering the sexually dimorphic pattern of zebrafish brain aging (Arslan-Ergul and Adams, 2014; Arslan-Ergul et al., 2016), care was taken to mix both males and females within each experimental group. All animal experiments were approved by the KU Leuven Animal Ethics Committee and executed in strict accordance with the European Communities Council Directive of 20 October 2010 (2010/63/EU).

\subsection{Optic nerve crush}

ON injury was performed as previously described (Bhumika et al., 2015; Lemmens et al., 2016; Zou et al., 2013). Briefly, zebrafish were anesthetized in $0.02 \%$ buffered tricaine (MS-222, Sigma
Aldrich). Sterile forceps (Dumont No. 5, FST) were used to remove the connective tissue surrounding the left eye, allowing to lift the eyeball out of its orbit and expose the ON. By carefully placing the sterile forceps around the (left) ON while avoiding damage to the ophthalmic artery, a crush of 10 seconds at $500 \mu \mathrm{m}$ distance of the ON head was performed, which was kept fixed at all ages and fish sizes. Of note, bilateral ON lesions were used to evaluate optokinetic responses (Elsaeidi et al., 2014; Zou et al., 2013). Independent naive and/or sham-operated eyes from separate fish were used as control, in agreement with recent publications reporting contralateral effects of eye/ON injuries (Gallego et al., 2012; Neve et al., 2012). As sham-operated eyes always showed similar results as compared to the uninjured situation, we used naive controls in most of the experiments further on.

\subsection{Tracing and quantification of tectal (re)innervation}

(Re)innervation of the optic tectum was assessed in naive and injured fish, sacrificed at 7 and $14 \mathrm{dpi}$, using biocytin as an anterograde tracer (Fig. S1), as previously described (Bhumika et al., 2015; Lemmens et al., 2016). Hereto, fish were anesthetized in $0.02 \%$ buffered tricaine and placed under a dissection microscope (Leica). After removal of connective tissue, the eye was lifted upwards, the ON was cut between the ON head and the site of crush, and a gelfoam with the dissolved tracer was placed between the 2 nerve ends. The fish were revived for 3 hours to allow anterograde transport of the tracer along the RGC axons, after which they were ultimately euthanized in $0.1 \%$ buffered tricaine and transcardially perfused with phosphate-buffered saline (PBS) and $4 \%$ paraformaldehyde (PFA). Subsequently, the brains were dissected, postfixated overnight in 4\% PFA, and embedded in $4 \%$ agarose in PBS. Serial coronal vibratome sections of $50 \mu \mathrm{m}$ were made and transferred to netwells (Corning). Biocytin, taken up by the regenerated axons, was visualized by means of the Vectastain $\mathrm{ABC}$ kit (Vectastain Laboratories) using diaminobenzidine as a chromogen. Brain sections were then mounted on glass slides and counterstained with nuclear red, in order to identify the sections containing the central optic tectum via the presence of specific nuclei (Wulliman et al., 1996). Imaging was performed at $10 \times$ using a microscope Zeiss Imager Z1. Tectal (re)innervation was quantified via an in-house-developed ImageJ script, in which the biocytinlabeled area was measured using a preset threshold. Next, axonal density was defined as the ratio of the biocytin positive $(+)$ area to the area innervated by the RGC axons, being the stratum fibrosum et griseum superficiale and the stratum opticum of the optic tectum. Tectal (re)innervation was evaluated in 5 to 8 fish per experimental group on at minimum 4 sections, containing the central optic tectum, per fish (Wulliman et al., 1996). Importantly, axonal density in naive young adults was considered maximal and set as a $100 \%$ reference value. (Re)innervation values of all other conditions were expressed relative to this reference value.

\subsection{Optokinetic response test}

To evaluate visual recovery after ON injury, adult zebrafish were subjected to an optokinetic response test at various time points after bilateral ONC, more specifically at 1, 3, 5, 7, 9, 11, 13, 16, 18, 20, 23, 25, 27, and 32 dpi. Per age group, 6 fishes were thus followed up over a consecutive period of 32 days. Fish were anesthetized briefly in $0.02 \%$ buffered tricaine to enable positioning in a custom-made glass chamber. Next, a continuous water flow was provided, reawakening the fish and allowing them to breathe while immobilized. The flow-through chamber was then placed in an OptoMotry device (Cerebral Mechanics) for visual stimulation (Mueller and Neuhauss, 2010). Visual acuity was assessed by determining 
the maximal spatial frequency of the fish, while velocity and contrast were kept fixed at $10 \mathrm{deg} / \mathrm{s}$ and $100 \%$, respectively. After an adaptation period of 40 seconds, each trial was initiated with a spatial frequency of 0.02 (c/d), which increased stepwise following a staircase model.

\subsection{Visualization and analysis of axonal elongation}

Early outgrowth and elongation of RGC axons were assessed at 2, 3,4 , and 5 days after ONC by visualization of regrowing RGC axons in horizontal visual system sections of $\mathrm{Tg}$ (Gap-43:eGFP) fish (Diekmann et al., 2015; Udvadia et al., 2001). Thereto, fish were euthanized in $0.1 \%$ buffered tricaine and transcardially perfused with PBS and 4\% PFA. The complete visual system as a whole was dissected, fixed overnight, and processed for horizontal cryosectioning (10- $\mu \mathrm{m}$ sections). Imaging of sections was carried out using an Olympus FV1000 confocal microscope at $20 \times$. The distance grown by the Gap- $43^{+}$axons, being the length between the crush site and the axonal growth tips, was determined on a minimum of 4 sections containing the complete $\mathrm{ON}$ tract and averaged per fish. Five to 6 fishes per experimental group were used.

\subsection{Tracing and quantification of the number of regenerated RGCS}

To evaluate the number of regenerating RGCs, we used again biocytin tracing, this time to visualize retrograde transport towards the regenerating RGCs (Fig. S1). Either naive or injured (2, 3, 4, or 7 dpi) fish were sedated ( $0.02 \%$ buffered tricaine), and the left ON was then exposed and cut right behind the crush site. A gelfoam with the dissolved tracer was applied on the proximal nerve end and the eye was placed back into its socket. Fish were revived for 3 hours, allowing the tracer to be retrogradely transported and label the RGC cell bodies in the retina. After 3 hours, the fish were euthanized in $0.1 \%$ buffered tricaine, the left eye was dissected and fixed overnight in 4\% PFA, and then processed for serial sagittal cryosectioning (10- $\mu \mathrm{m}$ sections). Biocytin was visualized using Alexa-488-coupled streptavidin (1:200, Invitrogen), and fluorescent imaging of the retina was performed using an Olympus FV1000 confocal microscope at $20 \times$. Biocytin ${ }^{+}$cells were counted on 6 central retinal crosssections at 80,160 , and $240 \mu \mathrm{m}$ distance at either side of the ON head and averaged per fish. Experimental groups included 3 to 5 fish.

\subsection{Retinal explant assay}

To assess the outgrowth potential of young and aged RGCs in vitro, retinal explants were made, according to previously published methods (Veldman et al., 2007). Three days after ONC, the retinas of 3 young and 3 aged injured fish were extracted, and explants of $400 \mu \mathrm{m}^{2}$ were created using a Tissue Chopper (McI1 wain Tissue Chopper, The Mickle Laboratory Engineering Co. Ltd). After digestion and rinsing, all as previously described (Veldman et al., 2007), the explants were cultivated, 1 retina per well, in a precoated 6-well plate. The explants were allowed to grow for 24 hours in a humidified, ambient air incubator at $28{ }^{\circ} \mathrm{C}$ and then fixed in $4 \%$ PFA. To avoid variation in neurite outgrowth between the central and peripheral retina, only explants of the central retina were cultivated. Eight explants extracted from young fish and 4 explants extracted from aged fish showed neurite outgrowth and were subsequently analyzed. Neurites were visualized via immunohistochemistry, using mouse anti- $\alpha$-tubulin (1:300, Sigma Aldrich, T9026) and Alexa-488 conjugated secondary antibody (1:200, Invitrogen) for detection. The explant body was stained using $4^{\prime}, 6-$ diamidino-2-phenylindole. Explants were then imaged with an Olympus FV1000 confocal microscope at $4 \times$. Neurite outgrowth was analyzed with a previously published custom script (Gaublomme et al., 2014), which makes use of a size selection tool to extract neurites attached to the explant body. As such, the total neurite area was measured and categorized into segments by drawing concentric circles with increasing diameter from the explant body: 0-100 $\mu \mathrm{m}$, $100-200 \mu \mathrm{m}, 200-300 \mu \mathrm{m}$, and more than $300 \mu \mathrm{m}$ from the explant edge, which enables to evaluate neurite elongation.

\subsection{Analysis of RGC cell death}

Cell death was visualized on $10 \mu \mathrm{m}$ cryosections of the left eye, processed as described previously, harvested from naive and injured ( $1 \mathrm{dpi}$ ) young and aged fish. After application of rabbit antiactivated caspase-3 (1:70, Biovision, 3015-100) and detection via the TSA FT/ Cy3 System (PerkinElmer), imaging of the stained sections was performed using an Olympus FV1000 confocal microscope at $20 \times$. Four fishes were used per experimental condition, and for each fish, 6 sections were qualitatively analyzed. Rabbit antiactivated caspase3 is commonly used as a marker for apoptosis and has already been applied on retinal sections of zebrafish to detect cell death (Bhumika et al., 2015; Lemmens et al., 2016). As a positive control, we standardly included slices of postnatal mouse cerebellum (Van Hove et al., 2012; Verslegers et al., 2013) and dimethyl sulfoxide-injected zebrafish eyes (Lemmens et al., 2016), all as described previously.

\section{9. $R N A$ isolation and quantitative real-time polymerase chain reaction}

Quantitative real-time polymerase chain reaction (PCR) was performed to assess the expression of gap43 and tuba1a in retinal tissue of naive and injured ( 3 and $7 \mathrm{dpi}$ ) young and aged fish. Whole retinas were dissected, pooled per 3 fishes, and homogenized in Tri reagent (Sigma-Aldrich). Total RNA was extracted by NucleoSpin RNA isolation kit (Machery-Nagel, Germany) and RNA was reverse transcribed to cDNA using oligo dT primers and Superscript III reverse transcriptase (Invitrogen, Belgium). The quantitative PCR reactions were performed using SYBR Green master mix (Applied Biosystems) and a StepOne Plus Real Time PCR system (Applied Biosystems). All reactions were run in triplicate and 3 to 5 independent samples (consisting of pooled retinas from 3 fishes each) were analyzed per experimental condition. Using GeNorm (qBase software (Vandesompele et al., 2002)), hypoxanthine phosphoribosyl-transferase 1 (hprt1) and succinate dehydrogenase complex subunit A flavoprotein (sdha) were selected as reference genes. Analysis of gene expression levels was performed using qBase software, which is based on the $\Delta \Delta$ Ct quantification method (Hellemans et al., 2007). Primer specificity (Table 1) was confirmed via dissociation curve analysis at the end of each qPCR reaction.

\subsection{Visualization and analysis of the innate immune response}

To visualize the inflammatory response upon axonal injury, $\mathrm{Tg}$ (coro1a:eGFP) reporter fish were used, in which microglia/

Table 1

Primers used for qPCR analysis

\begin{tabular}{lll}
\hline Gene & Primer sequence $\left(5^{\prime}->3^{\prime}\right)$ & Reference \\
\hline gap43 & F: CAGCCGACGTGCCTGAA & McCurley and Callard, 2010 \\
& R: TCCTCAGCAGCGTCTGGTTT & \\
tuba1 & F: GGAGCTCATTGACCTTGTTTAGATA & McCurley and Callard, 2010 \\
& R: GCTGTGGAAGACCAGGAAACC & \\
hprt1 & F: TGGACCGAACTGAACGTCTG & Bhumika et al., 2015 \\
& R: TGGGAATGGAGCGATCACTG & \\
Sdha & F: ACGCACCCAATGCCAAAGAC & \\
& R: TCTTTATCCGGCCCAACACC & \\
\hline
\end{tabular}

Key: F, forward; qPCR, quantitative polymerase chain reaction; $R$, reverse. 
macrophages appear green (Zou et al., 2013). Fish were euthanized at baseline or at defined time points postinjury $(4,7,14,21$, and 28 dpi) in $0.1 \%$ buffered tricaine, and their eyes were extracted. Eyes were then fixed for 1 hour in 4\% PFA. The retinas were dissected and postfixed in 4\% PFA, again for 1 hour. Next, the retinal whole mounts were stained with mouse anti-GFP (1:250, Millipore, MAB3580), using an Alexa-488 conjugated secondary antibody (1:200, Invitrogen). Imaging was performed with an Olympus FV1000 confocal microscope at $20 \times$. The number and distribution of inflammatory cells were evaluated using a recently published ImageJ script, combined with spatial statistical techniques (Davis et al., 2017). Global innate immune cell density was calculated by dividing the number of Coro1 $\mathrm{a}^{+}$cells by the total retinal area. In order to reveal possible differences in migration patterns between young and aged fish, innate immune cells were plotted as a function of distance, that is, the Euclidean distance of each microglia/ macrophage centroid, relative to the center of the ON head was determined. Results are presented as probability distribution functions, wherein the data were averaged per treatment group en normalized to young adult, naive baseline values. Data represent retinal analyses of 4 to 7 fishes per condition.

\subsection{Experimental design and statistical analysis}

Data were always analyzed by 2 blinded observers. All raw data were first tested for normality and homoscedasticity, and these assumptions were met in all cases. As such, comparisons were performed using the parametric Student's $t$ test, analysis of covariance, and 1- or 2-way analysis of variance followed by the Tukey post hoc test. All values are represented as mean \pm standard error of the mean, and $p \leq 0.05$ was considered statistically significant.

\section{Results}

\subsection{Tectal reinnervation after axonal injury is delayed upon aging}

To study the impact of aging on CNS regeneration, reinnervation of the optic tectum, quantified via anterograde biocytin tracing of the regenerating RGC axons (Fig. S1), was evaluated after ONC injury in fish of $5,12,18,24$, and 36 months old (Fig. S2). First, it is worth mentioning that zebrafish growth already coincides with small changes in naive axonal density values (Fig. 1A). Seven days after axonal injury to the ON, reinnervation of the tectum progressively reduces with aging and is significantly declined in 24$(64.3 \% \pm 5.3 \%)$ and $36-(58.1 \% \pm 6.3 \%)$ month-old fish, when compared to young adults ( $86.7 \% \pm 5.9 \%$ at 5 months of age). By 14 $\mathrm{dpi}$, however, tectal reinnervation is restored at all ages, suggesting the reinnervation process to be only delayed (Fig. 1A). Importantly, we anticipated a possible influence of fish size on this read-out, as it is well established that teleosts display an indeterminate growth through life span, whereby also the size of the visual system increases with aging (Johns and Easter, 1977; Mack et al., 2004). Regrowth of the injured axons within the ON may therefore take longer in larger, aged fish, where they have to traverse a greater distance. Nonetheless, analysis of covariance clearly shows the increase in reinnervation latency to be age specific and independent of fish size.

\subsection{Functional recovery after axonal injury is delayed in aged fish}

In order to confirm the observed age-related reinnervation latency, an optokinetic response test was used to evaluate functional recovery, which generally correlates well with tectal reinnervation by the RGC axons (Kaneda et al., 2008). Visual acuity was measured by determining the maximal spatial frequency eliciting an eye response of the fish and assessed at regular time points after bilateral ONC injury in 5- (young adults) and 24- (aged) month-old fish (Fig. 1B). Notably, sight is preserved with aging (maximal spatial frequency of $0.302 \pm 0.013 \mathrm{c} / \mathrm{d}$ in young vs. $0.355 \pm 0.024 \mathrm{c} /$ $\mathrm{d}$ in aged fish). Immediately after damage to the $\mathrm{ON}$, eyesight is completely lost, but it slowly recovers over time. In young adults, primary vision already starts to regain at $3 \mathrm{dpi}(0.007 \pm 0.007 \mathrm{c} / \mathrm{d})$ and visual acuity is restored to naive values by $16 \mathrm{dpi}(0.302 \pm 0.018$ $\mathrm{c} / \mathrm{d}$ ). However, aged fish only start retrieving vision from $7 \mathrm{dpi}$ on $(0.027 \pm 0.019 \mathrm{c} / \mathrm{d})$ and achieve complete visual recovery beyond 20 days after ONC $(0.340 \pm 0.009 \mathrm{c} / \mathrm{d})$. Although aged zebrafish are thus able to functionally recover from axonal injury, these results confirm an age-dependent delay in the $\mathrm{ON}$ regeneration process.

\subsection{Axonal outgrowth is decelerated in aged fish}

As tectal reinnervation and visual recovery were found to be delayed upon aging, we further investigated axonal outgrowth and
A

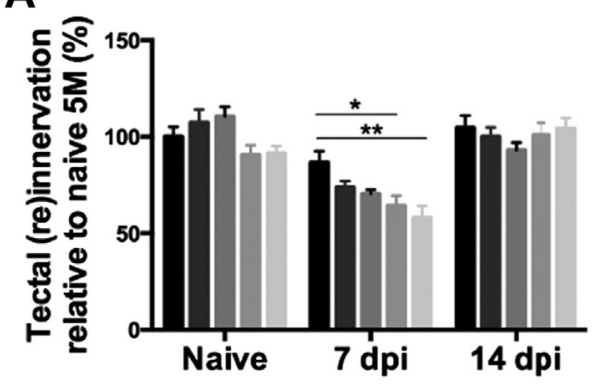

B

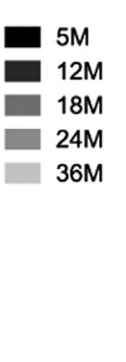

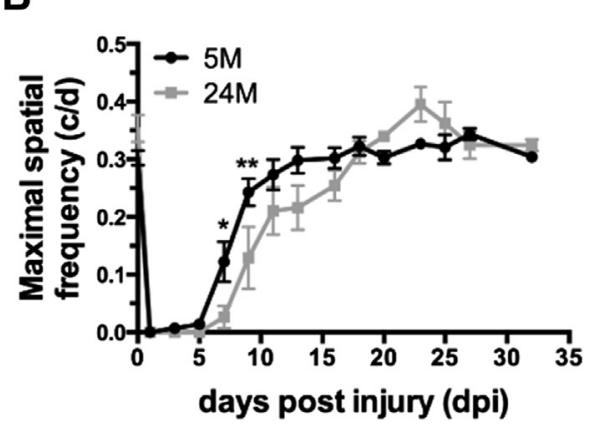

Fig. 1. Optic nerve regeneration is delayed in aged zebrafish. (A) Reinnervation of the optic tectum, quantified as the axonal density within the stratum fibrosum et griseum superficiale and the stratum opticum after anterograde biocytin tracing of the regenerating retinal ganglion cell axons, is delayed upon axonal injury in aged fish. At 7 days postinjury (dpi) to the optic nerve, tectal reinnervation is significantly reduced in 24- and 36-month-old fish, compared to young adults of 5 months old (ANOVA, $p=0.002$, $\mathrm{F}=$ 4.555. Tukey post hoc, ${ }^{*} p<0.05,{ }^{* *} p<0.01$ ), and the observed differences in axonal density are independent of fish size (ANCOVA, $p=0.577, \mathrm{~F}=0.347$ ). By 14 dpi, all fish have restored axonal density to naive age-matched values, indicating that tectal reinnervation by the RGC axons is merely decelerated in aged fish. Values are means \pm SEM, $\mathrm{n}=5-8$ animals. (B) Visual recovery was evaluated by determining the maximal spatial frequency eliciting an optokinetic response. While young adult fish already show return of this primary reflex from $3 \mathrm{dpi}$, aged individuals only start regaining sight at $7 \mathrm{dpi}$. Full restoration to naive visual acuity is achieved by 16 dpi in young adults, yet only by 20 dpi in aged fish. As such, aged fish show significantly lower maximal spatial frequency values at 7 and 9 dpi $(2$-way ANOVA, $p=0.068, \mathrm{~F}=3.370)$. Tukey post hoc, ${ }^{*} p<0.05,{ }^{* *} p<0.01$. Values are represented as mean $\pm S E M, n=6$ animals. Abbreviations: ANCOVA, analysis of covariance; ANOVA, analysis of variance; SEM, standard error of the mean. 
elongation. As growth-associated protein 43 (Gap-43) is known to be expressed in outgrowing axons (Holohan, 2015), regrowth of the injured axons was visualized within horizontal visual system sections of $\mathrm{Tg}$ (Gap-43:eGFP) fish (Fig. 2A). The distance regrown by the axons was assessed at different time points after ONC, which enabled to estimate growth rate over time (Fig. 2B). Our results show that at 2 dpi most axons are still situated at the level of the crush site. One day later, a substantial amount of axons has crossed the site of crush; yet, the number of regenerating axons is clearly reduced in aged fish, when compared to young adults. Moreover, the distance regrown by these axons comprises only $60 \%(444.15 \pm 104.54 \mu \mathrm{m})$ of that in young adults $(740.72 \pm 41.83 \mu \mathrm{m})$ at $3 \mathrm{dpi}$, indicating that aging significantly impacts axonal outgrowth initiation (Fig. 2B). By 5 dpi, the RGC axons are still, although slightly, behind of those in young adults. As demonstrated previously, aged fish are thus able to successfully regenerate upon ON injury, but early RGC outgrowth is clearly decelerated with aging. Next, the number of regenerating RGCs was quantified using a retrograde biocytin tracing paradigm (Fig. S1 and 2A). The number of retrogradely labeled RGCs is similar in naive fish of 5- and 24-month-old (Fig. 2C). However, and as expected, aged fish consistently display a lower number of regenerating RGCs after crush, which is already visible from 2 dpi on and lasts over time. Nevertheless, at $7 \mathrm{dpi}$, the number of biocytin ${ }^{+}$cells is equal to that in age-matched naive fish, indicating all RGCs are eventually able to regenerate. Taken together, our data reveal an important impact of aging on axonal outgrowth initiation, reflected by an age-related, transient reduction in the number of regenerating RGCs and their early (out)growth capacity.
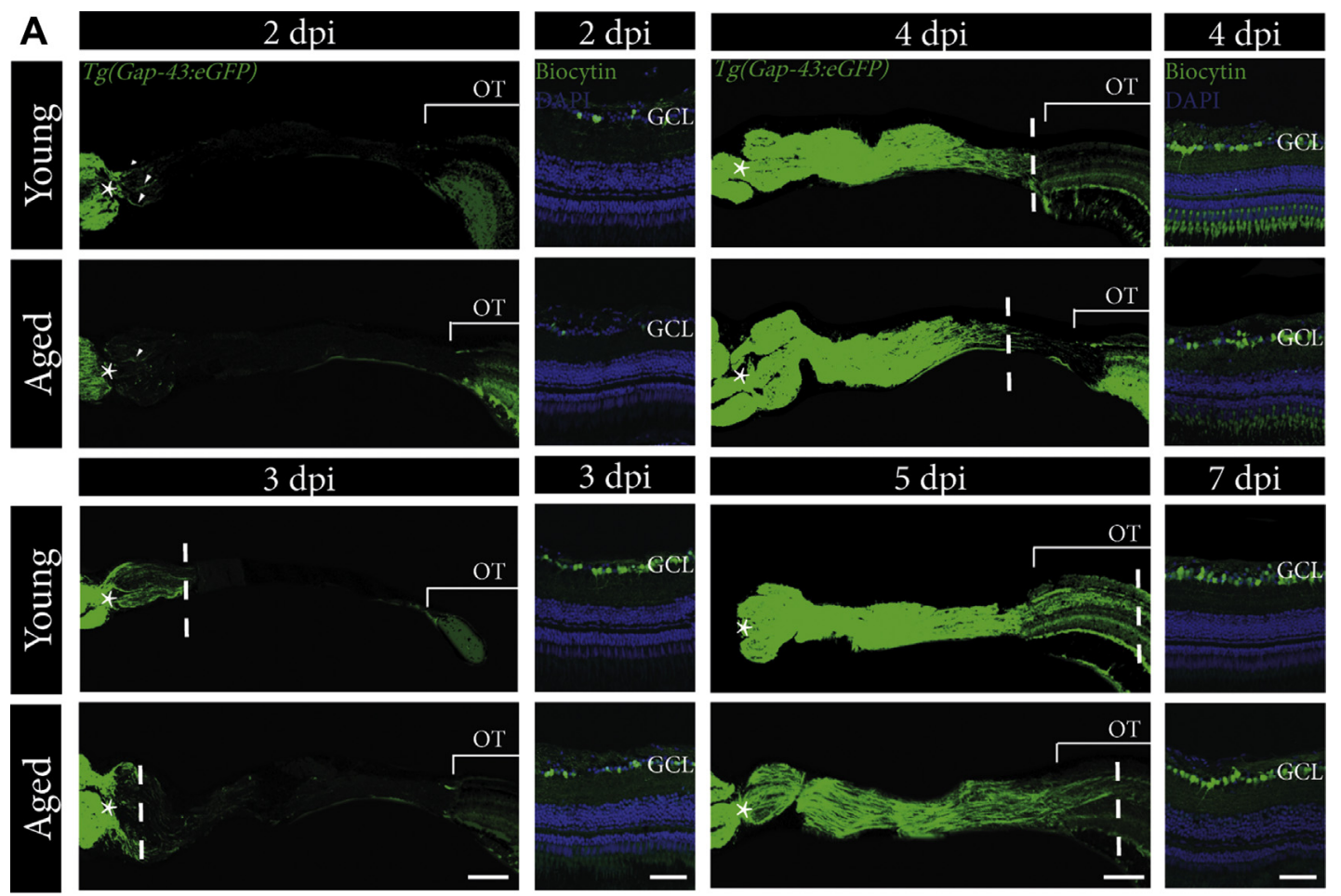

B

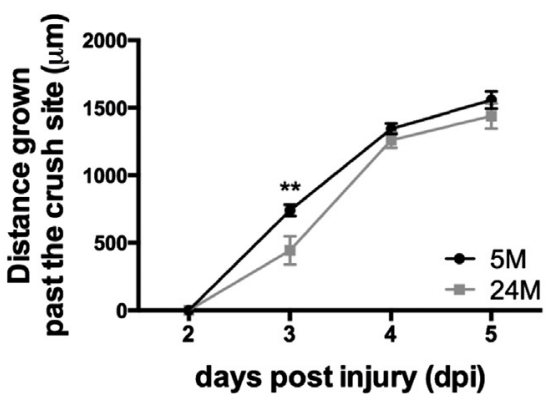

C

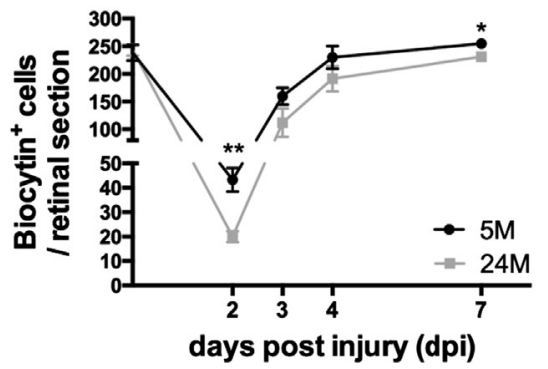

Fig. 2. Initiation and early retinal ganglion cell (RGC) axonal outgrowth are decelerated in aged fish. (A) Left panels: regrowing RGC axons were visualized in horizontal visual system sections of young (5 months) and aged (24 months) $\mathrm{Tg}$ (Gap-43:eGFP) fish. At 2 days postinjury (dpi), only a few pioneer RGCs have regrown an axon (white arrows), while most regrowing axons are still situated at the site of crush (indicated by ${ }^{*}$ ) in both young adult and aged fish. One day later, a significant amount of RGCs has already regrown an axon past the crush site in young adults, while outgrowth is still negligible in aged fish (dotted line indicating the growth front). By 4 and 5 dpi, axons reach the optic tectum (OT), being only slightly behind in distance at that time in aged fish, when compared to their younger counterparts. Scale bar $=200 \mu \mathrm{m}$. Right panels: The number of regenerating RGCs, regrowing an axon past the site of crush, was quantified after retrograde tracing with biocytin (Fig. S1). Already at 2 dpi, aged fish possess fewer regenerating RGCs when compared to young adults, but differences become less obvious toward later time points after injury. Scale bar $=50 \mu \mathrm{m}$. (B) Morphometric analysis of regrowing RGC axons in young (5M) and aged (24M) $\operatorname{Tg}$ (Gap-43:eGFP) fish uncovers an age-related delay in outgrowth initiation at 3 dpi (Tukey post hoc, ${ }^{* *} p<0.01$ ) and reveals that the average distance regrown by the RGC axons is consistently smaller in aged compared to young adults (2-way ANOVA, $p=0.004, F=9.304$ ). Values are means $\pm S E M$, $n=5-6$ animals. (C) Quantification of the number of regenerating RGCs, as visualized on retinal sections after retrograde biocytin tracing (Fig. S1), shows that the number of biocytin ${ }^{+}$RGCs per retinal section is smaller in aged fish, when compared to young adults, starting from 2 dpi on (2-way ANOVA, $p=0.031, \mathrm{~F}=5.196$. Tukey post hoc, ${ }^{*} p<0.05,{ }^{* *} p<0.01$ ). Values are means $\pm \mathrm{SEM}, \mathrm{n}=3-5$ animals. Abbreviations: ANOVA, analysis of variance; GCL, ganglion cell layer; SEM, standard error of the mean. 
3.4. The age-related decline in axonal outgrowth potential is recapitulated in an ex vivo retinal explant model

To further support the observed age-related decline in axonal outgrowth potential, growth onset and progression were assessed in an ex vivo setting, wherein retinal explants from young and aged injured fish were cultivated for 24 hours, and outgrowing neurites were subsequently stained for $\alpha$-tubulin. Our results demonstrate that the total area covered by neurites is drastically reduced in aged retinal explants, as compared to explants of young adult fish (Fig. 3A and B), thereby affirming a reduction in early outgrowth capacity with aging. In addition, also axon elongation appears disturbed, as explants from aged fish did not display neurites above $300 \mu \mathrm{m}$ of length. Overall, these data thus confirm the age-related decline in axonal outgrowth. Moreover, since external factors are absent in this ex vivo assay, these results indicate that an important part of the defects in axonal regeneration onset and progression are either intrinsic and/or the result of alterations in the immediate environment of the cells.

\subsection{All RGCs are able to survive axonal injury, even upon aging}

Although our results show that even in aged fish all RGCs are eventually able to regenerate after ON injury, the occurrence of cell death early in the process may contribute to a delay in RGC outgrowth initiation. As such, apoptosis was evaluated at $1 \mathrm{dpi}$, using immunohistochemistry for activated caspase-3 on retinal sections. As previously described in young adult fish, we do not detect RGC death after ONC (Zou et al., 2013), as no apoptotic cells are detected in the ganglion cell layer of both young and aged fish (Fig. S3). These results prove that, even upon aging, all RGCs are able to survive early after ONC injury.

\subsection{Aging results in a diminished intrinsic growth potential}

Although aged RGCs are not more vulnerable to cell death after injury to the $\mathrm{ON}$, a diminution in their intrinsic growth potential could possibly underlie the decline in early RGC outgrowth. Therefore, intrinsic neuronal growth capacity was evaluated by determining naive and injury-associated retinal mRNA expression levels of growth-associated genes gap-43 and tuba1a. Quantitative real-time PCR on retinal tissue of young and aged fish shows that the baseline expression of both genes is significantly reduced in aged retinas (gap-43: $1.00 \pm 0.15$ in young adults vs. $0.16 \pm 0.02$ in aged fish; tuba1a: $1.00 \pm 0.21$ in young adults vs. $0.29 \pm 0.13$ in aged fish; Fig. 4). Furthermore, mRNA levels remain significantly lower upon ONC in aged fish, even though axonal damage results in a clear upregulation in the expression of both genes, as in young adults. As such, both the naive and the injury-associated expression of growth-associated genes are curtailed in the aged zebrafish retina, suggesting an age-related decline in intrinsic neuronal growth capacity.

\subsection{Aging leads to a deceleration of the extrinsic inflammatory response}

Next, the potential effect of an aged cellular environment on the diminished regenerative potential in old fish was investigated. In light of the importance of inflammation for neural repair (Benowitz and Popovich, 2011; Bollaerts et al., 2017; Kyritsis et al., 2014), the innate immune response upon ONC was studied in young and aged $\mathrm{Tg}$ (coro1a:eGFP) fish-a reporter line expressing green fluorescent protein-labeled microglia and macrophages (Fig. 5A). As already previously reported (Zou et al., 2013), our morphometric analyses of retinal microglia/macrophages show that ON injury in young adults is followed by an increase in the number of innate immune cells, which peaks around $7 \mathrm{dpi}$, before returning to naive values around 28 days after the ONC (Fig. 5B). With aging, we reveal inflammaging to be apparent in the zebrafish retina, as the microglia density is higher compared to young adults $\left(792.15 \pm 42.31 \mathrm{cells} / \mathrm{mm}^{2}\right.$ in young adults vs. $910.08 \pm 32.28$ in aged fish). Moreover, in contrast to the swift innate immune response in young adults, aged individuals only reach a maximal increase in microglial/macrophage number around $21 \mathrm{dpi}$ (Fig. 5B). In addition, analysis of innate immune cell localization within the entire retina illustrates an injury-associated redistribution of the cell population toward the center of the retina (Fig. 5C). Importantly, in young adults most microglia/macrophages are situated at the center of the retina around 7 dpi (Fig. 5D). However, in aged fish the innate immune cells only redistribute around $14 \mathrm{dpi}$. Thus, consistent with the observed age-dependent delay in axonal regeneration, the inflammatory response upon $\mathrm{ON}$ injury appears decelerated with aging.

\section{Discussion}

For many neurodegenerative diseases, age is the main risk factor, and over the past years, it has become increasingly clear that the aging context of many neuropathologies provides an additional hurdle in translating basic neuroregenerative research carried out in young animal models to the clinical situation. Indeed, while animal models have forecasted the success of hundreds of neuroprotective and regenerative pharmacological treatments, almost without exception, all have failed in clinical trials (Johnson, 2015, 2016). As such, well-characterized gerontology models, wherein
A

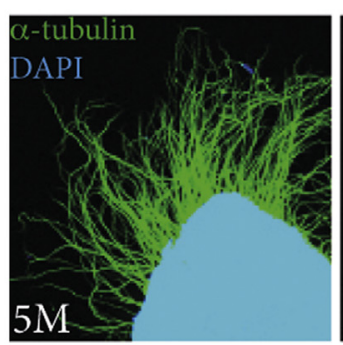

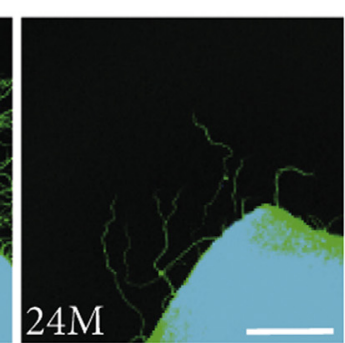

B

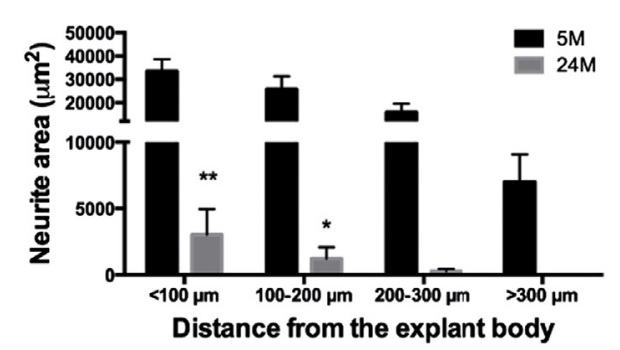

Fig. 3. The ex vivo axonal growth potential is declined in aged zebrafish. (A) Immunostainings for $\alpha$-tubulin on retinal explants of young ( 5 months) and aged (24 months) fish expose a clear decline in neurite outgrowth with aging. Scale bar $=200 \mu \mathrm{m}$. (B) Quantification of the immunodetected neurite outgrowth area, at $100-\mu \mathrm{m}$ increments from the explant body, shows that total neurite outgrowth is reduced within explants of aged fish and also that axonal extension is impaired. Explants of aged fish have consistently less neurites at all distances analyzed and do not possess neurites longer than $300 \mu \mathrm{m}$, as assessed 24 hours postcultivation ( 2 -way ANOVA, $p<0.0001$, $\mathrm{F}=20.940$. Tukey post hoc, $\left.{ }^{*} p<0.05,{ }^{* *} p<0.01\right)$. Values are means \pm SEM, $\mathrm{n}=4-8$ explants. Abbreviations: ANOVA, analysis of variance; SEM, standard error of the mean. 
gap-43

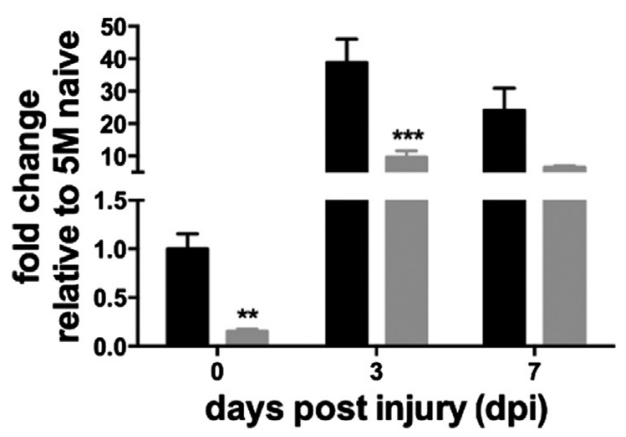

tuba1a

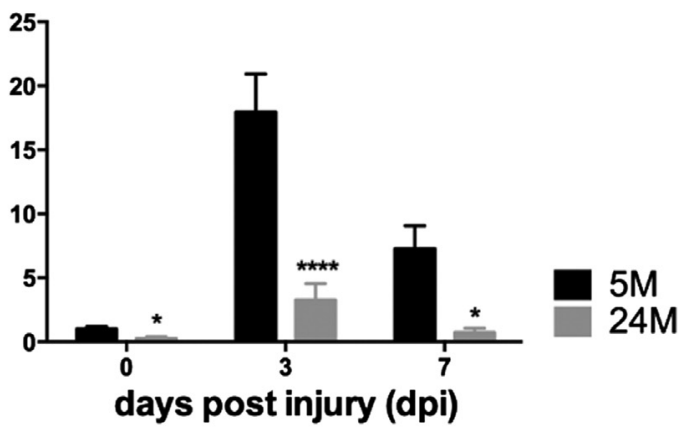

Fig. 4. Aged retinal ganglion cells (RGCs) show a reduced outgrowth capacity. Real-time PCR of the growth-associated genes gap-43 and tuba1a reveals a significant decline in naive expression levels with aging (gap-43: Student's $t$ test, $p=0.010, \mathrm{~F}=146.472$, tuba1a: Student's $t$ test, $p=0.023, \mathrm{~F}=3.835$ ). Also injury response levels are significantly reduced after optic nerve crush in aged fish (24 months), when compared to young adults (5 months; gap-43: 2-way ANOVA, $p=0.001, \mathrm{~F}=14.710$. tuba1a: 2-way ANOVA, $p<0.0001, \mathrm{~F}=53.650$. Tukey post hoc, $\left.{ }^{*} p<0.05,{ }^{* *} p<0.01,{ }^{* * *} p<0.001,{ }^{* * * *} p<0.0001\right)$. Values are means \pm SEM, $\mathrm{n}=3-5$ samples. Abbreviations: ANOVA, analysis of variance; Dpi $=$ days postinjury; SEM, standard error of the mean.

the influence of aging processes on neurodegeneration/regeneration can be adequately studied, will be indispensable in resolving age-related disease and/or damage in the mammalian CNS. Of course, considering that regenerative capacities are already limited in mammalian adulthood, researching neuroregeneration in the aging nervous system is notoriously difficult. Indeed, only recently, Geoffroy et al. (2016) revealed that after spinal cord lesion in mice, the growth-promoting effects of phosphatase and tensin homolog deletion are greatly diminished with aging, thereby representing the first study investigating the impact of aging on axonal regeneration in the mammalian CNS (Geoffroy et al., 2016). However, elucidating the etiology of age-related regenerative decline might be less challenging in a vertebrate animal model with high regenerative abilities.

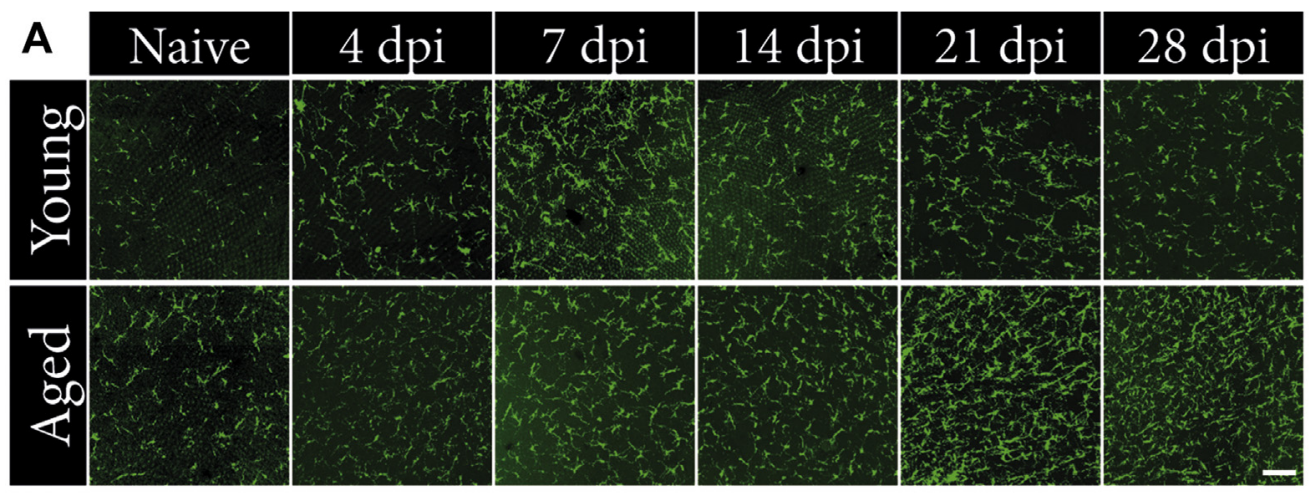

B

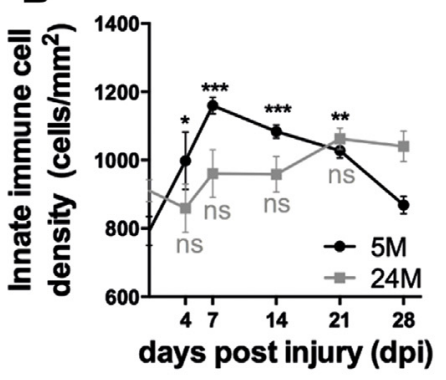

C

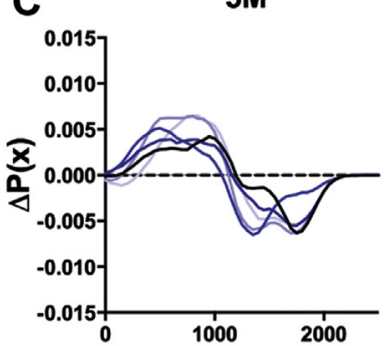

24M

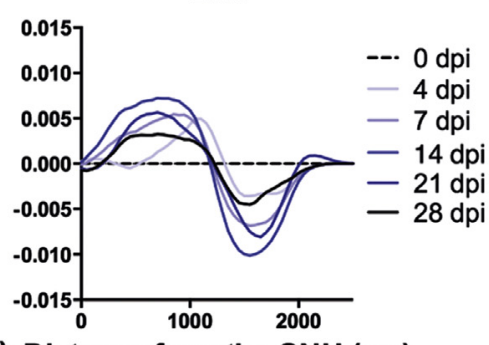

D

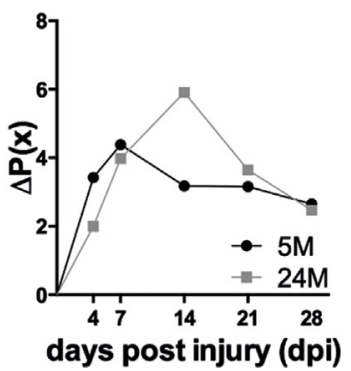

Fig. 5. The innate immune response is decelerated upon optic nerve injury in aged fish. (A) Insets of retinal whole mounts of $T g(C o r o 1 a: e G F P)$ fish, wherein microglia and macrophage appear green, illustrate an age-related retardation in the inflammatory response upon axonal injury. Scale bar $=50 \mu \mathrm{m}$. (B) Inflammaging is apparent in the senescent zebrafish retina, as innate immune cell density is significantly higher in naive aged fish ( 24 months), compared to young adults ( 5 months; Student's $t$ test, $p=0.039, \mathrm{~F}=1.861$ ). Upon optic nerve damage, young adults show a fast and significant rise in immune cell number, which already peaks around 7 days postinjury (dpi) $(2$-way ANOVA, $p<0,0001, \mathrm{~F}=$ 6340. Tukey post hoc, ${ }^{*} p<0.05,{ }^{* *} p<0.01,{ }^{* * *} p<0.001$ ). In contrast, microglia/macrophage density only peaks around $21 \mathrm{dpi}$ in aged fish, while this increase in immune cell number is still not significantly different from age-matched naive. Values are means $\pm S E M, n=4-7$ animals. (C) Detailed analyses of immune cell probability distributions in relation to the distance from the optic nerve head show the chance of finding a higher (positive values) or a lower (negative values) number of innate immune cells at a specific location in the retina, when compared to the naive situation (dotted line). This reveals a redistribution of the immune cell population from the periphery (defined as the area above $1000 \mu \mathrm{m}$ distance from the optic nerve head) towards the center of the retina (defined as the area below $1000 \mu \mathrm{m}$ distance from the optic nerve head) upon axonal injury in both young and aged fish (optic nerve crush [ONC] curves in blue and gray). (D) Starting from the probability distribution graphs (C), the area under every curve of each treatment group was calculated. This shows that immune cells migrate in response to ONC and accumulate at the center of the retina around 7 dpi in young fish and around 14 dpi in aged fish. Abbreviations: ANOVA, analysis of variance; ONH, optic nerve head; SEM, standard error of the mean. (For interpretation of the references to color in this figure legend, the reader is referred to the Web version of this article.) 
The current study addresses the axonal regeneration capacity of the aged zebrafish visual system, which proves to retain a remarkable regenerative ability. Although we did not yet investigate synaptic refinement and the restoration of retinotopy-which only occur 1-3 months after ONC in young adult zebrafish (Becker and Becker, 2008; Kaneda et al., 2008; McCurley and Callard, 2010; Zou et al., 2013), we here show that aged fish are capable of complete reinnervation of the optic tectum and successful visual recovery after ON injury. Nevertheless, we find these processes to be slowed upon aging, which is in line with findings in the goldfish spinal cord, where age likewise delays functional recovery (Bernstein, 1964). Further analysis revealed the observed agerelated retardation in the $\mathrm{ON}$ regeneration process to be most likely the result of a delay in the initiation of the regenerative response, as was also described in the peripheral nervous system of aged zebrafish (Graciarena et al., 2014). Indeed, we show aged zebrafish initially display fewer regenerating axons with less extensive outgrowth after injury, findings which are in agreement with those reported in the aged, regeneration-induced mammalian CNS (Geoffroy et al., 2016). Importantly, these age-dependent effects on early RGC outgrowth could be repeated in a retinal explant model, thereby confirming ex vivo retinal explant data from the young and aged frog visual system (Taylor et al., 1989). Moreover, as this ex vivo assay does not include several external factors, such as the presence of myelin-associated proteins and/or navigation cues, they do not seem to exert a major impact on the diminished RGC regeneration capacities in aged fish. As such, in search for the mechanisms that could underlie the observed age-related delay in zebrafish ON regeneration, we focused on intrinsic processes such as RGC viability and intrinsic growth capacity on the one hand, and extrinsic factors within their close environment, that is, the injury response of glial cells, on the other.

The cellular and extracellular changes that accompany aging render neurons vulnerable (Mattson and Magnus, 2006). Accordingly, 2-year-old mice show a faster time course of RGC loss following ON injury than mice of only 2 months (Wang et al., 2007). Fish, however, normally show no or very limited RGC death upon axonal injury (Zhou and Wang, 2002; Zou et al., 2013), as also confirmed by our data in young adults. Furthermore, our results indicate that also aged zebrafish RGCs are able to survive ON injury, as apoptosis is absent from the ganglion cell layer of both young and aged fish at 1 day after ONC. While our data thus suggest that aging does not lead to an increased susceptibility of zebrafish RGCs (at least early upon axonal injury), we do show their intrinsic growth potential to be clearly impacted by age. In mammals, both Gap-43 and $\alpha$-tubulin expression levels are often found to be reduced in the aged CNS, associated with an overall decline in synaptic plasticity and memory (Casoli et al., 1996, 2004; Riascos et al., 2014; Vanguilder and Freeman, 2011; Zhang et al., 2015). Here, we uncover a similar aging phenotype in the zebrafish, in which the baseline expression levels of both growth-associated genes are diminished with age. Since in naive zebrafish, Gap-43 expression is mainly situated in the RGC dendrites (Diekmann et al., 2015), most likely supporting continuous remodeling, the age-related decline in Gap-43 expression may well indicate reduced synaptic plasticity and intrinsic neuronal aging. More importantly, also the injuryassociated expression levels of both genes are significantly curtailed in aged fish. Indeed, although aged zebrafish do show a clear upregulation in the expression of both gap-43 and tuba1a, they do not reach the same expression levels as compared to young adults. In line with these results, one study already reported a gradual agedependent loss of a sustained Gap-43 response upon seizureinduced synaptic plasticity in the rat hippocampus (Schmoll et al., 2005). More aging research is certainly needed here, but generally the induction of growth-associated genes is well known to be of major importance for successful regeneration. Indeed, overexpression of Gap-43 is known to enhance mouse spinal cord regeneration (Bomze et al., 2001), while its downregulation hampers the generation of reactive sprouts upon climbing fiber axotomy (Allegra Mascaro et al., 2013). Taken together, we hypothesize that intrinsic neuronal aging of the RGCs, reflected by the agedependent decrease in gap-43 and tuba1a expression, results in the insufficient upregulation of these genes (and possibly other growth-associated factors) upon injury, and that this may lead to the observed delay in outgrowth initiation, and an overall retardation of the axonal regeneration process. Yet, the actual source of the age-related decrease in zebrafish axonal growth capacity remains to be elucidated, as besides neuronal aging of the RGCs themselves, also changes in their extracellular environment, for example, in growth factor and/or cytokine signaling can influence the growth potential.

Indeed, also macroglia and microglia undergo morphological and functional changes with age, known as cellular senescence. Furthermore, aged microglia alter their secretion pattern in favor of proinflammatory cytokines, leading to a chronic low-grade inflammation status, a phenomenon called "inflammaging." The aged mammalian CNS also displays a clear increase in microglial number (Damani et al., 2011; Streit et al., 2014). This, however, does not implicate increased neuroprotection but is assumed to compensate for the diminished activity of individual aged microglia. Notably, also acute responses to injury seem age dependent, as senescent microglia show a reduced mobilization to the injury site while their resolution is retarded (Damani et al., 2011; Wasserman et al., 2008). Increasing evidence for a detrimental effect of inflammaging on the regenerative outcome is emerging but has only been scarcely described in fish. In a model of ON remyelination (Munzel et al., 2014), decreased recruitment of microglia/macrophages to the site of injury was suggested to underlie regeneration failure in 15- to 18-month-old fish, as the early rise in innate immune cell number seen in young adults appeared blunted upon aging. Likewise, our data demonstrate the manifestation of inflammaging in the aged zebrafish retina, characterized not only by an increase in naive microglial number with aging but also a clear absence of an early inflammatory response upon axonal injury. Indeed, aged fish show a decelerated increase in innate immune cell density, and their inflammatory response upon ON injury is still not resolved by $28 \mathrm{dpi}$, in contrast to young adults. In addition, our data illustrate an injury-associated shift in innate immune cell location from the periphery toward the center of the retina. Possibly, this reflects a migration/attraction of immune cells, most probably comprising both proliferating resident microglia and infiltrating macrophages, towards the site of injury, which thus occurs significantly later in aged fish. As such, we are currently also addressing the timing of the innate immune response within the $\mathrm{ON}$ to unravel the cellular fingerprint of neuroglial interactions at the site of ONC. In addition, it will be most interesting to elucidate if the resolution of these immune cells is likewise delayed with aging, as is described after focal laser injury in the retina of aged mice (Damani et al., 2011). Indeed, a quick resolution of microglia and/or macrophages after injury could be of vital importance for successful regeneration, as stimulating the inflammatory response via intravitreal injection of zymosan, a yeast cell wall glucan, has been shown to promote RGC survival and regeneration early upon ON transection in young adult fish, while resulting in RGC cell death after 14 days (Zou et al., 2013). Also in the visual system of adult rats, the timing of zymosan delivery was reported to be of vital importance in the stimulation of axonal regrowth (Yin et al., 2003). 
Altogether, the immune response upon ONC injury appears deregulated in aged zebrafish. However, at this point, we can only speculate about the underlying mechanisms that link a timed immune response to successful ON regeneration in young adults and about the implications of aberrant inflammation on the regenerative process. Fish microglia have mainly been attributed a phagocytic role in neuronal repair, removing debris from cells that have undergone apoptosis or dismantling damaged axons (Colavincenzo and Levine, 2000; Zupanc et al., 2003). Yet, as our results show microglia/macrophages remain present in the retina for several days in young adults-at the time that RGC axonal regeneration is fully ongoing-we hypothesize that they exert a supportive function in ON regeneration, in agreement with the study described previously showing a transitory, yet regenerationpromoting effect of inflammatory stimulation early upon ON injury in zebrafish (Bollaerts et al., 2017; Zou et al., 2013). Also in goldfish, tumor growth factor- $\beta 1$ has been implicated as a growthsupportive factor derived from granular macrophages during ON regeneration (Battisti et al., 1995). Finally, in the injured zebrafish brain, inflammation has been proposed to beneficially affect neuroregeneration and promote neurogenesis by activating injuryinduced molecular programs, such as cysteinyl leukotriene signaling (Kyritsis et al., 2012). Future studies addressing microglial/macrophage activation, polarization state, and cytokine expression profile-a largely unexplored area in zebrafish regeneration research-are undoubtedly needed and will be of utmost importance to elucidate the exact function of inflammation/ inflammaging in successful CNS regeneration.

\section{Conclusions}

Our findings show that aged fish are able to functionally recovery from axonal injury in the CNS. However, despite the conservation of robust regenerative capacities, both intrinsic as well as extrinsic factors may impact the normally flawless ON regeneration process and could underlie the observed agerelated decline in regeneration onset and early outgrowth of axons. Further in-depth research into the molecules underlying neuronal aging and immune senescence will certainly provide novel insights and may contribute to the identification of innovative therapeutic targets to stimulate regeneration in an aging environment.

\section{Disclosure statement}

The authors have no actual or potential conflicts of interest.

\section{Acknowledgements}

The authors thank Professor Dr Ava Udvadia, University of Winsconsin, for the kind gift of the $\operatorname{Tg}$ (Gap-43:eGFP) fish line. They are grateful to Lut Noterdaeme and Marijke Christiaens for their technical laboratory support. This work has been funded by research grants from KU Leuven (BOF-OT14/00830) and the Research Foundation Flanders (FWO-Vlaanderen, Belgium, G0B2315N). JVH and EG are supported by the Flemish government agency for Innovation by Science and Technology (IWT-Vlaanderen, Belgium), and IB holds an FWO PhD fellowship. AB is supported by L'oreal-unesco "For women in science" (FWO-Vlaanderen, Belgium). BMD is supported by the Welcome Trust Healthcare Innovation Challenge fund. IVH, KL, and LDG are postdoctoral fellows funded by the Research foundation Flanders and KU Leuven.

\section{Appendix A. Supplementary data}

Supplementary data associated with this article can be found, in the online version, at http://dx.doi.org/10.1016/j.neurobiolaging. 2017.08.013.

\section{References}

Allegra Mascaro, A.L., Cesare, P., Sacconi, L., Grasselli, G., Mandolesi, G., Maco, B. Knott, G.W., Huang, L., De Paola, V., Strata, P., Pavone, F.S., 2013. In vivo single branch axotomy induces GAP-43-dependent sprouting and synaptic remodeling in cerebellar cortex. Proc. Natl. Acad. Sci. U. S. A. 110, 10824-10829.

Arslan-Ergul, A., Adams, M.M., 2014. Gene expression changes in aging zebrafish (Danio rerio) brains are sexually dimorphic. BMC Neurosci. 15, 29.

Arslan-Ergul, A., Erbaba, B., Karoglu, E.T., Halim, D.O., Adams, M.M., 2016. Shortterm dietary restriction in old zebrafish changes cell senescence mechanisms. Neuroscience 334, 64-75.

Battisti, W.P., Wang, J., Bozek, K., Murray, M., 1995. Macrophages, microglia, and astrocytes are rapidly activated after crush injury of the goldfish optic nerve: a light and electron microscopic analysis. J. Comp. Neurol. 354, 306-320.

Becker, C.G., Becker, T., 2008. Adult zebrafish as a model for successful central nervous system regeneration. Restorative Neurol. Neurosci. 26, 71-80.

Becker, T., Becker, C.G., 2014. Axonal regeneration in zebrafish. Curr. Opin. Neurobiol. 27, 186-191.

Benowitz, L.I., Popovich, P.G., 2011. Inflammation and axon regeneration. Curr. Opin. Neurol. 24, 577-583.

Bernstein, J.J., 1964. Relation of spinal cord regeneration to age in adult goldfish. Exp. Neurol. 9, 161-174.

Bhumika, S., Lemmens, K., Vancamp, P., Moons, L., Darras, V.M., 2015. Decreased thyroid hormone signaling accelerates the reinnervation of the optic tectum following optic nerve crush in adult zebrafish. Mol. Cell Neurosci. 68, 92-102.

Bollaerts, I., Van houcke, J., Andries, L., De Groef, L., Moons, L., 2017. Neuroinflammation as Fuel for axonal regeneration in the injured vertebrate central nervous system. Mediators Inflamm. 2017, 14.

Bomze, H.M., Bulsara, K.R., Iskandar, B.J., Caroni, P., Skene, J.H., 2001. Spinal axon regeneration evoked by replacing two growth cone proteins in adult neurons. Nat. Neurosci. 4, 38-43.

Casoli, T., Di Stefano, G., Delfino, A., Fattoretti, P., Bertoni-Freddari, C., 2004. Vitamin E deficiency and aging effect on expression levels of GAP-43 and MAP-2 in selected areas of the brain. Ann. New York Acad. Sci. 1019, 37-40.

Casoli, T., Spagna, C., Fattoretti, P., Gesuita, R., Bertoni-Freddari, C., 1996. Neuronal plasticity in aging: a quantitative immunohistochemical study of GAP-43 distribution in discrete regions of the rat brain. Brain Res. 714, 111-117.

Colavincenzo, J., Levine, R.L., 2000. Myelin debris clearance during Wallerian degeneration in the goldfish visual system. J. Neurosci. Res. 59, 47-62.

Damani, M.R., Zhao, L., Fontainhas, A.M., Amaral, J., Fariss, R.N., Wong, W.T., 2011. Age-related alterations in the dynamic behavior of microglia. Aging Cell 10, 263-276.

Davis, M.B., Salinas-Navarro, M., Cordeiro, F., Moons, L., De Groef, L., 2017. Characterizing microglia activation: a spatial statistics approach to maximize information extraction. Scientific Rep. 7, 1576.

Diekmann, H., Kalbhen, P., Fischer, D., 2015. Characterization of optic nerve regeneration using transgenic zebrafish. Front. Cell Neurosci. 9, 118.

Edelmann, K., Glashauser, L., Sprungala, S., Hesl, B., Fritschle, M., Ninkovic, J., Godinho, L., Chapouton, P., 2013. Increased radial glia quiescence, decreased reactivation upon injury and unaltered neuroblast behavior underlie decreased neurogenesis in the aging zebrafish telencephalon. J. Comp. Neurol. 521, 3099-3115.

Elsaeidi, F., Bemben, M.A., Zhao, X.F., Goldman, D., 2014. Jak/Stat signaling stimulates zebrafish optic nerve regeneration and overcomes the inhibitory actions of Socs3 and Sfpq. J. Neurosci. 34, 2632-2644.

Fleisch, V.C., Fraser, B., Allison, W.T., 2011. Investigating regeneration and functional integration of CNS neurons: lessons from zebrafish genetics and other fish species. Biochim. Biophys. Acta 1812, 364-380.

Gallego, B.I., Salazar, J.J., de Hoz, R., Rojas, B., Ramirez, A.I., Salinas-Navarro, M., Ortin-Martinez, A., Valiente-Soriano, F.J., Aviles-Trigueros, M., VillegasPerez, M.P., Vidal-Sanz, M., Trivino, A., Ramirez, J.M., 2012. IOP induces upregulation of GFAP and MHC-II and microglia reactivity in mice retina contralateral to experimental glaucoma. J. Neuroinflammation 9, 92.

Gaublomme, D., Buyens, T., De Groef, L., Stakenborg, M., Janssens, E., Ingvarsen, S., Porse, A., Behrendt, N., Moons, L., 2014. Matrix metalloproteinase 2 and membrane type 1 matrix metalloproteinase co-regulate axonal outgrowth of mouse retinal ganglion cells. J. Neurochem. 129, 966-979.

Geoffroy, C.G., Hilton, B.J., Tetzlaff, W., Zheng, B., 2016. Evidence for an agedependent decline in axon regeneration in the adult mammalian central nervous system. Cell Rep. 15, 238-246.

Gerhard, G.S., 2003. Comparative aspects of zebrafish (Danio rerio) as a model for aging research. Exp. Gerontol. 38, 1333-1341.

Gerhard, G.S., Cheng, K.C., 2002. A call to fins! Zebrafish as a gerontological model. Aging Cell 1, 104-111.

Graciarena, M., Dambly-Chaudiere, C., Ghysen, A., 2014. Dynamics of axonal regeneration in adult and aging zebrafish reveal the promoting effect of a first lesion. Proc. Natl. Acad. Sci. U. S. A. 111, 1610-1615. 
Hellemans, J., Mortier, G., De Paepe, A., Speleman, F., Vandesompele, J., 2007. qBase relative quantification framework and software for management and automated analysis of real-time quantitative PCR data. Genome Biol. 8, R19.

Holohan, M.R., 2015. GAP-43 in synaptic plasticity: molecular perspectives. Res. Rep. Biochem. 5, 137-146.

Johns, P.R., Easter Jr., S.S., 1977. Growth of the adult goldfish eye. II. Increase in retinal cell number. J. Comp. Neurol. 176, 331-341.

Johnson, I.P., 2015. Age-related neurodegenerative disease research needs aging models. Front. Aging Neurosci. 7, 168.

Johnson, I.P., 2016. Response: Commentary: Age-related neurodegenerative disease research needs aging models. Front. Aging Neurosci. 8, 44.

Kaneda, M., Nagashima, M., Nunome, T., Muramatsu, T., Yamada, Y., Kubo, M., Muramoto, K., Matsukawa, T. Koriyama, Y. Sugitani, K., Vachkov, I.H., Mawatari, K., Kato, S., 2008. Changes of phospho-growth-associated protein 43 (phospho-GAP43) in the zebrafish retina after optic nerve injury: a long-term observation. Neurosci. Res. 61, 281-288.

Keller, E.T., Murtha, J.M., 2004. The use of mature zebrafish (Danio rerio) as a model for human aging and disease. Comp. Biochem. Physiol. Toxicol. Pharmacol. 138 335-341.

Kishi, S., 2011. The search for evolutionary developmental origins of aging in zebrafish: a novel intersection of developmental and senescence biology in the zebrafish model system. Birth Defects Res. C Embryo Today 93, 229-248.

Kishi, S., Uchiyama, J., Baughman, A.M., Goto, T., Lin, M.C., Tsai, S.B., 2003. The zebrafish as a vertebrate model of functional aging and very gradual senescence. Exp. Gerontol. 38, 777-786.

Kyritsis, N., Kizil, C., Brand, M., 2014. Neuroinflammation and central nervous system regeneration in vertebrates. Trends Cell Biol. 24, 128-135.

Kyritsis, N., Kizil, C., Zocher, S., Kroehne, V., Kaslin, J., Freudenreich, D., Iltzsche, A. Brand, M., 2012. Acute inflammation initiates the regenerative response in the adult zebrafish brain. Science 338, 1353-1356.

Lemmens, K., Bollaerts, I., Bhumika, S., De Groef, L., Van houcke, J., Darras, V.M., Van Hove, I., Moons, L., 2016. Matrix metalloproteinases as promising regulators of axonal regrowth in the injured adult zebrafish retinotectal system. J. Comp. Neurol. 524, 1472-1493.

Mack, A.F., Sussmann, C., Hirt, B., Wagner, H.J., 2004. Displaced amacrine cells disappear from the ganglion cell layer in the central retina of adult fish during growth. Invest. Ophthalmol. Vis. Sci. 45, 3749-3755.

Mattson, M.P., Magnus, T., 2006. Ageing and neuronal vulnerability. Nat. Rev. Neurosci. 7, 278-294.

McCurley, A.T., Callard, G.V., 2010. Time course analysis of gene expression patterns in zebrafish eye during optic nerve regeneration. J. Exp. Neurosci. 2010, 17-33.

Mueller, K.P., Neuhauss, S.C., 2010. Quantitative measurements of the optokinetic response in adult fish. J. Neurosci. Methods 186, 29-34.

Munzel, E.J., Becker, C.G., Becker, T., Williams, A., 2014. Zebrafish regenerate full thickness optic nerve myelin after demyelination, but this fails with increasing age. Acta Neuropathologica Commun. 2, 77.

Neve, L.D., Savage, A.A., Koke, J.R., Garcia, D.M., 2012. Activating transcription factor 3 and reactive astrocytes following optic nerve injury in zebrafish. Comp. Biochem. Physiol. Toxicol. Pharmacol. 155, 213-218.

Riascos, D., Nicholas, A., Samaeekia, R., Yukhananov, R., Mesulam, M.M., Bigio, E.H., Weintraub, S., Guo, L., Geula, C., 2014. Alterations of $\mathrm{Ca}(2)(+)$-responsive proteins within cholinergic neurons in aging and Alzheimer's disease. Neurobiol. Aging 35, 1325-1333.

Schmoll, H., Ramboiu, S., Platt, D., Herndon, J.G., Kessler, C., Popa-Wagner, A., 2005. Age influences the expression of GAP-43 in the rat hippocampus following seizure. Gerontology 51, 215-224.
Streit, W.J., Xue, Q.S., Tischer, J., Bechmann, I., 2014. Microglial pathology. Acta Neuropathologica Commun. 2, 142.

Taylor, J., Jack, J., Easter, S., 1989. Is the capacity for optic nerve regeneration related to continued retinal ganglion cell production in the frog? Eur. J. Neurosci. 1 626-638.

Udvadia, A.J., Koster, R.W., Skene, J.H., 2001. GAP-43 promoter elements in transgenic zebrafish reveal a difference in signals for axon growth during CNS development and regeneration. Development 128, 1175-1182.

Van houcke, J., De Groef, L., Dekeyster, E., Moons, L., 2015. The zebrafish as a gerontology model in nervous system aging, disease, and repair. Ageing Res. Rev. 24, 358-368.

Van Hove, I., Verslegers, M., Buyens, T., Delorme, N., Lemmens, K., Stroobants, S., Gantois, I., D’Hooge, R., Moons, L., 2012. An aberrant cerebellar development in mice lacking matrix metalloproteinase-3. Mol. Neurobiol. 45, 17-29.

Vandesompele, J., De Preter, K., Pattyn, F., Poppe, B., Van Roy, N., De Paepe, A., Speleman, F., 2002. Accurate normalization of real-time quantitative RT-PCR data by geometric averaging of multiple internal control genes. Genome Biol. 3. RESEARCH0034.

Vanguilder, H.D., Freeman, W.M., 2011. The hippocampal neuroproteome with aging and cognitive decline: past progress and future directions. Front. Aging Neurosci. 3,8 .

Veldman, M.B., Bemben, M.A. Thompson, R.C. Goldman, D., 2007. Gene expression analysis of zebrafish retinal ganglion cells during optic nerve regeneration identifies KLF6a and KLF7a as important regulators of axon regeneration. Developmental Biol. 312, 596-612.

Verslegers, M., Van Hove, I., Buyens, T., Dekeyster, E., Knevels, E., Moons, L., 2013. Identification of MMP-2 as a novel enhancer of cerebellar granule cell proliferation. Mol. Cell Neurosci. 57, 63-72.

Wang, A.L., Yuan, M., Neufeld, A.H., 2007. Age-related changes in neuronal susceptibility to damage: comparison of the retinal ganglion cells of young and old mice before and after optic nerve crush. Ann. New York Acad. Sci. 1097, 64-66.

Wang, Z., Jin, Y., 2011. Genetic dissection of axon regeneration. Curr. Opin. Neurobiol. 21, 189-196.

Wasserman, J.K., Yang, H., Schlichter, L.C., 2008. Glial responses, neuron death and lesion resolution after intracerebral hemorrhage in young vs. aged rats. Eur. J. Neurosci. 28, 1316-1328.

Westerfield, M., 2000. The Zebrafish Book. A Guide for the Laboratory Use of Zebrafish (Danio rerio), fourth ed. Univiversity of Oregon Press, Eugene.

Wulliman, M., Rupp, B., Reichert, H., 1996. Neuroanatomy of the Zebrafish Brain: A Topological Atlas. Birkhäuser, Basel.

Yin, Y., Cui, Q., Li, Y., Irwin, N., Fischer, D., Harvey, A.R., Benowitz, L.L, 2003. Macrophage-derived factors stimulate optic nerve regeneration. J. Neurosci. 23, 2284-2293.

Zhang, F., Su, B., Wang, C., Siedlak, S.L., Mondragon-Rodriguez, S., Lee, H.G., Wang, X., Perry, G., Zhu, X., 2015. Posttranslational modifications of alpha-tubulin in alzheimer disease. Translational Neurodegener. 4, 9.

Zhou, L.X., Wang, Z.R., 2002. Changes in number and distribution of retinal ganglion cells after optic nerve crush in zebrafish. Shi yan sheng wu xue bao 35, 159-162.

Zou, S., Tian, C., Ge, S., Hu, B., 2013. Neurogenesis of retinal ganglion cells is not essential to visual functional recovery after optic nerve injury in adult zebrafish. PLoS One 8, e57280.

Zupanc, G.K., Clint, S.C., Takimoto, N., Hughes, A.T., Wellbrock, U.M., Meissner, D., 2003. Spatio-temporal distribution of microglia/macrophages during regeneration in the cerebellum of adult teleost fish, Apteronotus leptorhynchus: a quantitative analysis. Brain Behav. Evol. 62, 31-42. 